\title{
THIẾT KẾ Bộ CẢM BIẾN Độ MẶN CỦA NƯớC SỬ DỤNG PHƯƠNG PHÁP ĐIỆN CUỤC KHÔNG TIẾP XÚC
}

\author{
Bùi Thư Cao ${ }^{1}$, Ong Mẫu Dũng ${ }^{1}$, Trần Mạnh Tiến ${ }^{2}$ \\ ${ }^{1}$ Khoa Công Nghệ Điện Tủ, Truòng Đại học Công nghiệp Thành phố Hồ Chí Minh, \\ ${ }^{2}$ Trung tâm Dịch vu Phân tỉch Thi nghiệm Thành phố Hồ Chi Minh; \\ buithucao@iuh.edu.vn
}

Tóm tắt. Máy đo độ mặn trực tuyến cho nước là một trong những thiết bị quan trọng nhất trong việc theo dõi và quản lý môi trường nước cho các trang trại nuôi trồng thủy sản. Điểm yếu của các thiết bị đo độ mặn hiện tại là sử dụng phương pháp tiếp xúc trực tiếp với môi trường nước thông qua màng trao đổi ion trong các điện cực cảm biến. Với phương pháp này, theo thời gian, các điện cực sẽ bị ố, rỉ sét và gây ra các lỗi lớn cho phép đo. Để khắc phục nhược điểm này, chúng tôi đề xuất đo độ mặn của nước bằng phương pháp điện cực không tiếp xúc. Phương pháp này sử dụng sóng vô tuyến để đo hằng số điện môi của môi trường nước. Sau đó, dựa trên mối quan hệ giữa độ mặn với hẳng số điện, độ mặn của nước được nội suy. Với phương pháp được đề xuất, ăng ten điện cực cảm biến được bọc cách điện bằng nhựa và nhúng trong môi trường nước. Do đó, hệ thống đo độ mặn được đề xuất không bị ảnh hưởng bởi vết bẩn và rỉ sét trong môi trường nước. Vì vậy, nó có độ bền và độ ổn định cao và có thể được sử dụng liên tục trong nhiều tháng mà không cần vệ sinh và bảo trì.

Từ khóa. Độ mặn; RF - Tần số vô tuyến, Hằng số điện môi.

\section{DESIGN SALINITY SENSOR USING NON-CONTACTING ELECTRODE METHOD}

\begin{abstract}
The online salinity meter for water is one of the most important devices in monitoring and managing water environment for aquaculture farms. The weakness of current salinity measurement devices is to use a method of directing contact with the water environment through ion exchange membranes in sensor electrodes. With this method, over time, the electrodes will be stained, rust and cause big errors for the measurement. To overcome this drawback, we propose to measure the salinity of water by contactless electrode method. This method uses radio waves to measure the dielectric constant of the water environment. Then, based on the relation between salinity with electrical constant, the water salinity is interpolated. With the proposed method, the sensor electrode antennas are coverd with plastic and embedded in water environment. Therefore, the proposed salinity measurement system is not affected by stainning and rusting in water environment. So, it has high durability and stability and can be used continuously months without cleanning and maintenance.
\end{abstract}

Keywords. Salinity; RF - Radio Frequency, Dielectric Constant.

\section{INTRODUCTION}

Trong việc nuôi trồng thủy sản để cho năng suất cao và hiệu quả thì việc giám sát các thông môi trường nước là vô cùng quan trọng. Một trong những thông số quan trọng đó là độ mặn của nước. Độ mặn của nước ảnh hưởng rẩt lớn đến quá trình sinh trưởng và năng suất của hầu hết các loại thủy, hải sản nước lợ và nước mặn. Hiện nay có ba cách xác định độ mặn phổ biến như [1].

\subsection{Phương pháp tỷ trọng kế (Hydrometer)}

Tỷ trọng là tỷ số của trọng lượng riêng chất cần đo và trọng lượng riêng của nước. Độ mặn được đo bằng cách đo sự thay đổi của tỷ trọng nước không có muối và tỷ trọng của nước khi được hoàn tan muối. Mặc dù độ mặn và tỉ trọng có quan hệ với nhau, nhưng hai thuật ngữ thì khá khác nhau và nên được hiểu theo nghĩa riêng của nó. Độ mặn: được định nghĩa là khối lượng muối được hòa tan trong $1 \mathrm{~kg}$ nước biển. Tỉ trọng: được định nghĩa là tỉ số giữa tỉ trọng của dung dịch (tại nhiệt độ nhất định) trên tỉ trọng của nước 
tinh khiết (tại nhiệt độ nhất định). Tỉ trọng phụ thuộc vào nhiệt độ, vì vậy muốn kết quả đo tỉ trọng đúng thì nhiệt độ phải đúng.

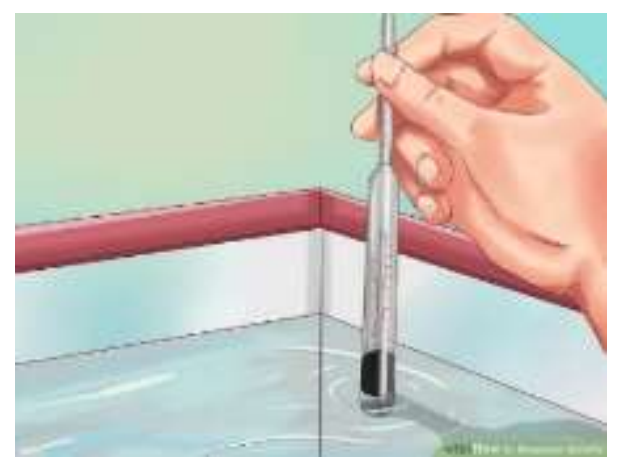

Hình 1. Thiết bị đo độ mặn bằng phuoong pháp đo tỷ trọng

\subsection{Phương pháp khúc xạ kế (Refractometer)}

Khúc xạ kế đo theo nguyên tắc sóng ánh sáng truyền qua các môi trường có chiết suất khác nhau sẽ có sự thay đổi phương truyền của tia sóng. Chiết xuất của nước liên quan đến nồng độ vật chất trong môi trường nước. Khi muối được hòa tan vào môi trường nước sẽ làm thay đổi nồng độ vật chất, dẫn đến thay đổi về hệ số khúc xạ của nước, như được thể hiện trong [2].

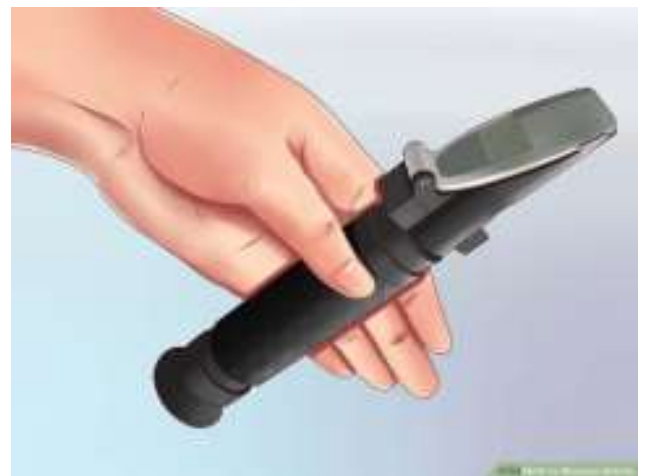

Hình 2. Thiết bị đo độ mặn bằng phuoong pháp khúc xạ kế

\subsection{Phương pháp độ dẫn điện (Electrical Conductivity)}

Dựa trên phương pháp đo độ dẫn điện $(\mathrm{EC})$ của dung dịch. Muối trong dung dịch chủ yếu tồn tại ở 2 dạng ion Sodium $(\mathrm{Na}+)$ và ion Chloride $(\mathrm{Cl}-)$. Khi số lượng ion Sodium và ion Chloride tăng lên, độ dẫn điện của dung dịch cũng tăng lên tương ứng với độ tăng của nồng độ muối. Sử dụng nguyên lý này, độ mặn được xác định bằng cách tính toán độ dẫn điện của môi trường qua mang trao đổi ion. Nó còn được gọi là phương pháp đo kỹ thuật số. Phương pháp EC được khuyến nghị sử dụng trong đo độ mặn trong nông nghiệp, như được minh họa qua thiết bị đo EC170.

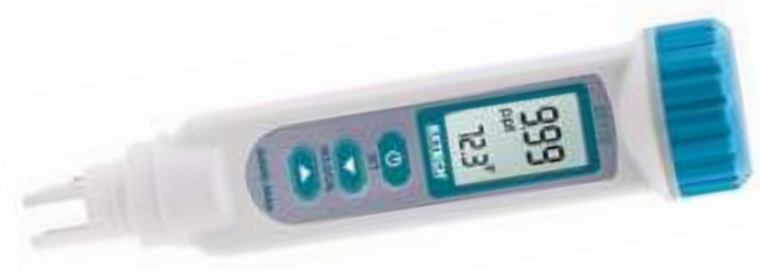

Hình 3. Máy đo độ dẫn EC170 của hãng EXTECH 


\subsection{Nhận xét chung}

Các phương pháp khúc xạ kế và tỷ trọng kế sử dụng thao tác nhân công để đo độ mặn. Điều này rất bất tiện khi giám sát các thông số môi trường trong các điều kiện nắng mưa hay trời tối. Với phương pháp độ dẫn điện, tuy có độ chính xác cao nhưng việc đo thực hiện qua tiếp xúc trực tiếp của điện cực đo qua mang trao đổi ion với môi trường nước. Phương pháp này có nhược điểm là nếu sử dụng đo online trong môi trường nước, theo thời gian các điện cực sẽ bị oxy hóa, bám bẩn, đóng váng từ môi trường. Điều này sẽ gây ra sai số lớn cho phép đo, ảnh hưởng đến chất lượng của hệ thống giám sát môi trường.

Để khắc phục nhược điểm này, nhóm nghiên cứu đề xuất đo độ mặn bằng phương pháp điện cực không tiếp xúc. Phương pháp này sử dụng bức xạ của sóng radio để đo hằng số điện môi của môi trường nước, từ đó nội suy ra độ mặn của nước.

\section{PROPOSED METHOD}

\subsection{Mô hình đề nghị cho thiết bị đo độ mặn}

Hệ thống đo độ mặn được đề nghị gồm 2 phần, phần phát và phần thu. Phần phát, tín hiệu nguồn $\mathrm{s}(\mathrm{t})$ từ mạch tạo xung clock với tần số $1 \mathrm{KHz}$ được điều chế với sóng mang từ bộ LCO (local oscillator), với tần số $315 \mathrm{MHz}$. Anten được thiết kế loại anten Helical. Tín hiệu sau điều chế được khuếch đại công suất qua bộ PA (power amplifier) rồi bức xạ ra anten phát. Phần thu, tín hiệu từ anten thu sẽ đi qua mạch cộng hưởng chọn lọc tần số sóng mang LC, sau đó được giải điều chế với sóng mang fc. Tín hiệu sau khi được giải điều chế được đi qua bộ khuếch đại công suất thấp LA (low-noise amplifier) và mạch lọc lowpass filter với băng thông $1 \mathrm{KHz}$, để khôi phục nguồn tín hiệu ban đầu s' (t). Bên cạnh đó hệ thống còn được thiết kế thêm bộ đo nhiệt độ dùng PT100. Sau đó các tín hiệu thu s' $(\mathrm{t})$ và $\mathrm{T}(\mathrm{t})$ được đưa đến bộ chuyển đổi thu thập dữ liệu số Data Acquisition, NI my DAQ. Dữ liệu số thu được đưa đến bộ xử lý số DSP để phân tích và tính toán giá trị độ mặn. Việc xử lý DSP và hiển thị kết quả được thực hiện trên máy tính với phần mềm LabView.

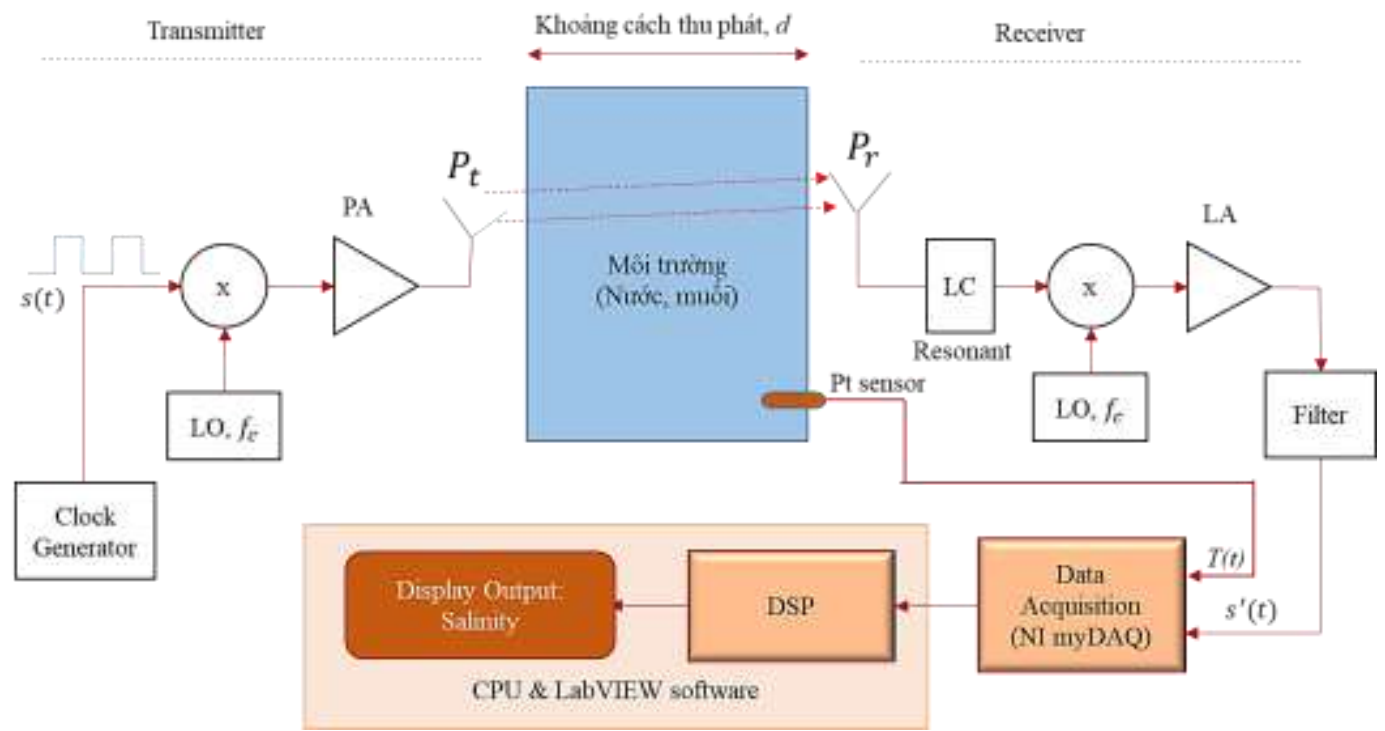

Hình 1. So đồ khối phần cứng của hệ thống máy đo độ mặn

\subsection{Phương pháp thiết kế}

\subsubsection{Xây dựng hàm toán học mô tả hệ thống thu phát}

Gọi $P_{t}$ là công suất phát của anten phát, $P_{r}$ là công suất thu được của anten thu. Trong đó anten phát và anten thu được thiết kế giống nhau Hertzian dipole, có cùng diện tích bực xạ hiệu dụng là $A_{e}$ và độ lợi được xây dựng từ [3],

$$
G=\frac{4 \pi A_{e}}{\lambda^{2}}
$$

Trong đó, $\lambda$ là bước sóng của sóng mang truyền dẫn trong môi trường vật chất, 


$$
\lambda=\frac{v_{p}}{f}
$$

Trong đó, $v_{p}$ là vận tốc truyền sóng trong môi trường vật chất,

$$
v_{p}=\sqrt{\frac{1}{\varepsilon \mu}}=\sqrt{\frac{1}{\varepsilon_{0} \varepsilon_{r} \mu_{0} \mu_{r}}}=\sqrt{\frac{1}{\varepsilon_{0} \mu_{0}}} \sqrt{\frac{1}{\varepsilon_{r} \mu_{r}}}=\frac{c}{\sqrt{\varepsilon_{r} \mu_{r}}}
$$

Trong đó, $\varepsilon_{r}$ là hằng số điện môi của môi trường truyền sóng, $\varepsilon_{0}$ là hệ số điện môi của môi trường truyền sóng không gian tự do, $\mu_{r}$ là hằng số từ môi của môi trường truyền sóng, $\mu_{0}$ là hệ số từ môi của môi trường truyền sóng không gian tự do.

$$
\begin{aligned}
& \varepsilon_{0}=8.854 \times 10^{-12} \mathrm{~F} / \mathrm{m} \\
& \mu_{o}=4 \pi \times 10-7 \underline{\mathrm{H}} / \underline{\mathrm{m}} \\
& \lambda_{0}=\frac{c}{f}
\end{aligned}
$$

Với $c=\sqrt{\frac{1}{\varepsilon_{0} \mu_{0}}}=3 \times 10^{8} \mathrm{~m} / \mathrm{s}$ và $f$ là tần số của sóng mang

Một cách tổng quát $\varepsilon_{r}$ là hằng số điện môi phức, $\varepsilon_{r}=\varepsilon_{r}^{\prime}-j \varepsilon_{r}^{\prime \prime}$. Trong môi trường nước biển, $\mu_{r} \approx 1$.

Từ (2), (3) và (6) ta có,

$$
\lambda=\frac{v_{p}}{f}=\frac{c}{f \sqrt{\varepsilon_{r} \mu_{r}}}=\frac{\lambda_{0}}{\sqrt{\varepsilon_{r}}}
$$

Công suất của anten thu [3] là,

$$
P_{r}=\frac{P_{t} G_{t} G_{r} \lambda^{2}}{(4 \pi d)^{2}}
$$

Với $G_{t}$ và $G_{r}$ là độ lợi của anten phát và thu, xem như giống nhau trong hệ thống anten đẳng hướng helical [4] có,

$$
G_{t}=G_{r} \approx 15\left(\frac{2 \pi R}{\lambda}\right)^{2} n\left(\frac{L}{\lambda}\right)=\frac{60(\pi R)^{2} n L}{\lambda^{3}}
$$

Với $\mathrm{R}$ là bán kính của vòng dây quấn anten là $2 \mathrm{~mm}$, L là khoảng cách giữa các vòng dây là $1.5 \mathrm{~mm}, \mathrm{n}$ là số vòng dây quấn và chiều dài của dây quấn là $47 \mathrm{~cm}$.

Vậy từ (7) và (8) ta có,

$$
P_{r}=\frac{225 \pi^{2} R^{4}(n L)^{2}}{d^{2} \lambda_{0}^{4}} P_{t} \varepsilon_{r}^{2}
$$

Mặt khác, theo lý thuyết bức xạ [4], ta có công suất phát của anten:

$$
P_{t}=\frac{Z k^{2}}{12 \pi}|I h|^{2}
$$

Với, $\mathrm{I}$ là dòng diện trên anten, $\mathrm{h}$ là độ dài của anten, $\mathrm{k}$ là hằng số truyền dẫn sóng,

Do hệ thống anten thu phát bọc cách điện nên $\sigma \approx 0$, ta có:

$$
k=\alpha+j \beta, \alpha=\omega \sqrt{\frac{\mu \varepsilon}{2}\left(\sqrt{1+\left(\frac{\sigma}{\omega \varepsilon}\right)^{2}}-1\right)}, \beta=\omega \sqrt{\frac{\mu \varepsilon}{2}\left(\sqrt{1+\left(\frac{\sigma}{\omega \varepsilon}\right)^{2}}+1\right)}
$$

$$
\begin{aligned}
& k=j \beta=j \omega \sqrt{\mu \varepsilon}=j \omega \sqrt{\mu_{0} \varepsilon_{0}} \sqrt{\mu_{r} \varepsilon_{r}}=\frac{j 2 \pi f}{c} \sqrt{\varepsilon_{r}}=\frac{j 2 \pi}{\lambda_{0}} \sqrt{\varepsilon_{r}} \\
& Z=\sqrt{\frac{\mu}{\varepsilon}}=\sqrt{\frac{\mu_{0} \mu_{r}}{\varepsilon_{0} \varepsilon_{r}}}=\frac{Z_{0}}{\sqrt{\varepsilon_{r}}}
\end{aligned}
$$

Với $Z_{0}$ là trở kháng sóng trong môi trường không gian tự do, $Z_{0} \approx 377 \Omega$

Vậy, $P_{t}=\frac{Z k^{2}}{12 \pi}|I h|^{2}=\frac{\pi Z_{0}(I h)^{2}}{3 \lambda_{0}^{2}} \varepsilon_{r}^{0.5}$

Từ (10) và (13) ta có,

$$
P_{r}=\frac{75 \pi^{3} R^{4}(n L)^{2} Z_{0}(I h)^{2}}{d^{2} \lambda_{0}^{6}} \varepsilon_{r}^{2.5}
$$

\subsubsection{Xây dựng hàm mô tả hằng số điện môi}

Độ mặn của nước biển phụ thuộc vào thành phần của các nguyên tố chính trong nước: Chloride, Sodium, Magnesium, Calcium và một số nguyên tố khác trong nước tại vùng địa phương, như được thể 
hiện trong [5] và [6] . Chính những thành phần chất này tạo ra sự thay đổi của hằng số điện môi của môi trường nước biển. Hằng số điện môi của môi trường vật chất được mô tả đầu tiên bởi Debye [7] năm 1929 ,

$$
\varepsilon_{r}=\varepsilon_{\infty}+\frac{\varepsilon_{S}-\varepsilon_{\infty}}{1+(j 2 \pi f \tau)^{1-\varnothing}}-j \frac{\delta}{2 \pi f \varepsilon_{0}}
$$

Trong đó, $\varepsilon_{\infty}$ là hằng số điện môi ở tần số cực cao của dung dịch, $\varepsilon_{S}$ là hằng số điện môi tỉnh (ở tần số thấp), $\tau$ là thời gian ổn định phân cực (relaxation time) của dung dịch, $\varnothing$ là thống số thực nghiệm (rất bé) và $\delta$ là độ dẫn điện ion.

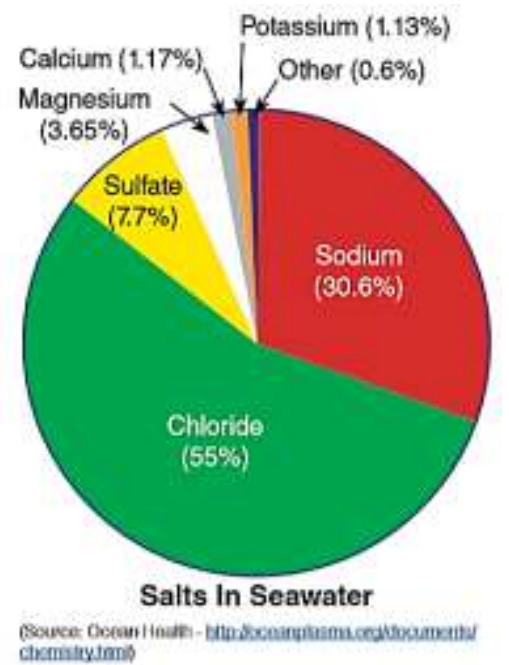

Hình 4. Thành phần cấu tạo của nuớc biển

Đến nay, đã có nhiều tác giả nghiên cứu xây dựng phương trình mô tả hằng số điện môi của dung dịch nước biển trong mối quan hệ với độ mặn, tần số sóng truyền dẫn như được review bởi Ram [8]. Nhìn chung các mô hình này đã xây dựng để hoàn thiện mô tả các thông số $\varepsilon_{\infty}, \varepsilon_{S}, \tau$ và $\delta$. Chúng là hàm số của độ mặn và nhiệt độ.

Gọi $\mathrm{T}$ là nhiệt độ $0 \mathrm{C}$ và $\mathrm{S}$ là độ mặn \%o của nước. Thông số $\varepsilon_{\infty}$ trong môi trường nước lợ là 4.9 , được đo trong nghiên cứu [9]. Hệ thống anten cách điện với môi trường, nên $\delta \approx 0$. Để đơn giản hóa bài toán thiết kế, phương pháp đề nghị chọn tần số làm việc tại $315 \mathrm{MHz}$. Giá trị của tham số $\tau$ theo cách tính [7] và [10] có giá trị rất bé, nhỏ hơn 17.6 nano giây. Do đó $(j 2 \pi f \tau)^{1-\emptyset}$, tính tại tần số làm việc là rất bé so với 1 , Nên ta xem như được bỏ qua $(j 2 \pi f \tau)^{1-\emptyset}$ trong pt $(15)$. Vậy $\varepsilon_{r}$ có giá trị thực và pt $(15)$ được viết gọn là,

$$
\varepsilon_{r}=\varepsilon_{S}(T, S)
$$

Qua khảo sát các nghiên cứu trước, chúng tôi chọn mô hình mô tả hằng số điện môi của Swith [7]. Vì theo nghiên cứu của Gadani 2012 [10], phương pháp của Swith được đánh giá là cho kết quả gần với thực nghiệm nhất. Theo Swith, hằng số điện môi được thể hiện là,

$$
\varepsilon_{r}=\varepsilon_{S}(T, S)=\varepsilon_{0}(T, 0) a(S, T)
$$
khiết,

Trong đó $\varepsilon_{0}(T, 0)$ là hàm mô tả hằng số điện môi với biến số nhiệt độ trong môi trường nước tinh

$$
\varepsilon_{0}(T, 0)=87.134-1.949 \times 10^{-1} T-1.276 \times 10^{-2} T^{2}+2.491 \times 10^{-4} T^{3}
$$

$a(S, T)$ là hàm đặc trưng biến thiên của hằng số điện môi theo nhiệt độ và độ mặn. Với phương pháp của Swith, hàm đặc trưng này được mô tả là,

$$
a(S, T)=1+1.613 \times 10^{-3} S T-3.656 \times 10^{-3} S+3.21 \times 10^{-5} S^{2}-4.322 \times 10^{-7} S^{3}
$$

\subsubsection{Phương pháp hiệu chuẩn công suất thu}

Từ pt (14) ta có,

$$
\begin{aligned}
& P_{r}=\frac{75 \pi^{3} R^{4}(n L)^{2} Z_{0}(I h)^{2}}{d^{2} \lambda_{0}^{6}} \varepsilon_{r}^{2.5}=P_{r 0} \varepsilon_{r}^{2.5} \\
& \text { Trong đó, gọi } P_{r 0}=\frac{75 \pi^{3} R^{4}(n L)^{2} Z_{0}(I h)^{2}}{d^{2} \lambda_{0}^{6}} .
\end{aligned}
$$


Vậy với một hệ thống anten thu phát được thiết kế cố định thì $P_{r 0}$ có giá trị được xác định và là hằng số. $P_{r 0}$ được tính dựa vào phép hiệu chuẩn công suất, được thực hiện ngay sau khi lắp đặt hệ thống tại địa điểm đo.

Thủ tục hiệu chuẩn công suất như sau:

1) Sử dụng máy đo độ mặn chuyên dụng EC170 của hãng Extech để đo độ nặm của môi trường nước tại thời điểm đó.

2) Từ pt (17), (18) và (19) ta tìm được $\varepsilon_{r}$ của nước.

3) Dựa vào hằng số điện môi đã tìn được và công suất thu được của anten thu vào pt (20) ta tìm ra được $P_{r 0}$.

\subsubsection{Phương pháp đo độ mặn}

Để đo độ mặn tại thời điểm bất kỳ, ta thực hiện các bước sau:

Bước 1) Đo công suất và nhiệt độ tại thời điểm đó bằng thiết bị đo độ mặn được thiết kế.

Bước 2) Tính $\varepsilon_{r}$ từ $\mathrm{pt}(20)$ và $\varepsilon_{0}(T, 0)$ từ pt $(18)$

Bước 3) Từ $\varepsilon_{r}, \varepsilon_{0}(T, 0)$ và pt $(17)$ ta tìm được giá trị của hàm đặc trưng $a(S, T)$

Bước 4) Dựa trên nhiệt độ đo được và giá trị của hàm đặc trưng $a(S, T)$, ta giải pt (19) bằng phương pháp Newton-Raphson, ta tìm ra được giá trị độ mặn.

Hìn 5. Giải thuật đo độ mặn

\section{RESULTS}

Hệ thống sau khi được lắp đặt như Hình 6 và Hình 7. Bể nước 500 lit, bán kính $50 \mathrm{~cm}$, chiều cao 70 $\mathrm{cm}$. Mô đun thu phát được lắp trong ống nhựa $\mathrm{PVC}$ đường kính $2.7 \mathrm{~cm}$. Đấu cuối anten có bọc nhựa, được tiếp xúc trực tiếp với môi trường nước ở độ sâu $30 \mathrm{~cm}$. Dây fide được bọc giáp và nối với mô đun thu thập dữ lion NI MyDAQ. Dữ liệu thu từ NI MyDAQ được truyền về máy tính qua cổng USB. Hình 8 . Minh họa giao diện phần mềm xử lý dữ liệu và nội suy kết quả độ mặn.

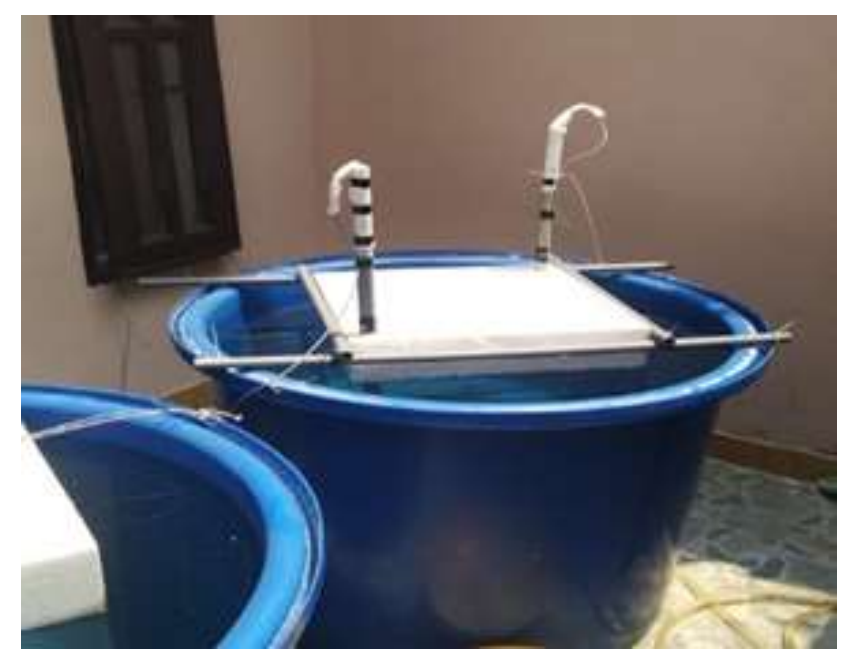

Hình 6. Bể nuớc muối và hệ thống anten thu phát được lắp đặt trong 02 ống nhựa 


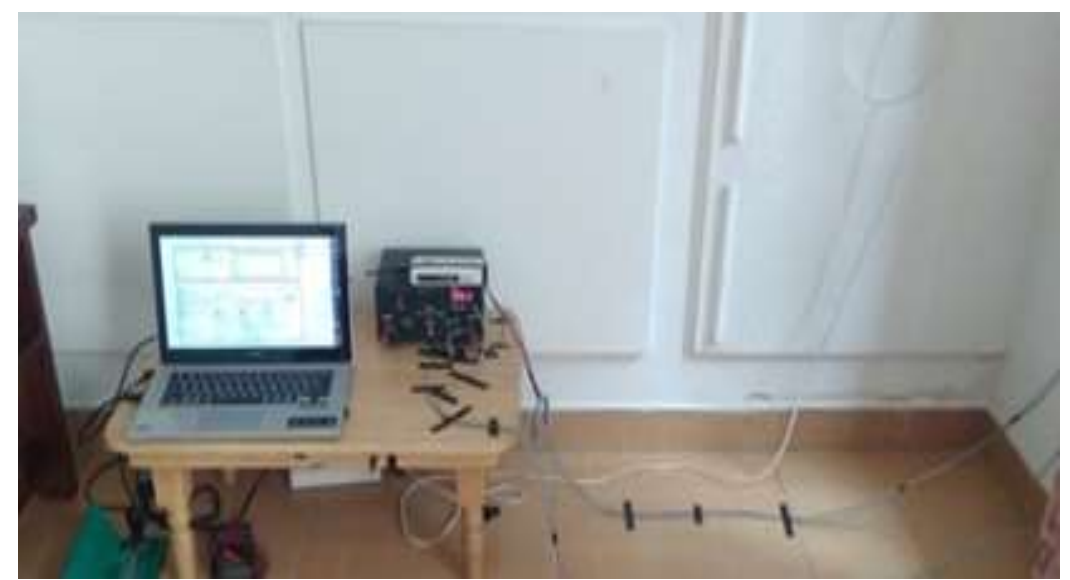

Hìn 7. Hệ thống thu thập và xủ lý dũ liệu

Các dữ liệu đo, ghi nhận và thống kê thời gian, như thể hiện trong Bảng 1 , Hình 9 và Hình 10 . Trong đó, ta ký hiệu: $\mathrm{S} 0 / 00$ là độ mặn của nước thực tại thời điểm đo, $\mathrm{S}$ EX $0 / 00$ là độ mặn đo bằng máy đo chuyên dụng EC170 của hãng EXTECH và Delta $(\mathrm{S})$ là sai số của phép đo. Việc hiệu chuẩn công suất tại thời điểm độ mặn bằng zero. Kết quả thực nghiệm cho thấy thiết bị và hệ thống đo độ mặn có sai số lớn hơn khi so sánh với máy đo khúc xạ kế và độ dẫn. Ta thây sai số gia tăng khi nhiệt độ môi trường tăng cao (khoảng trưa và chiều).

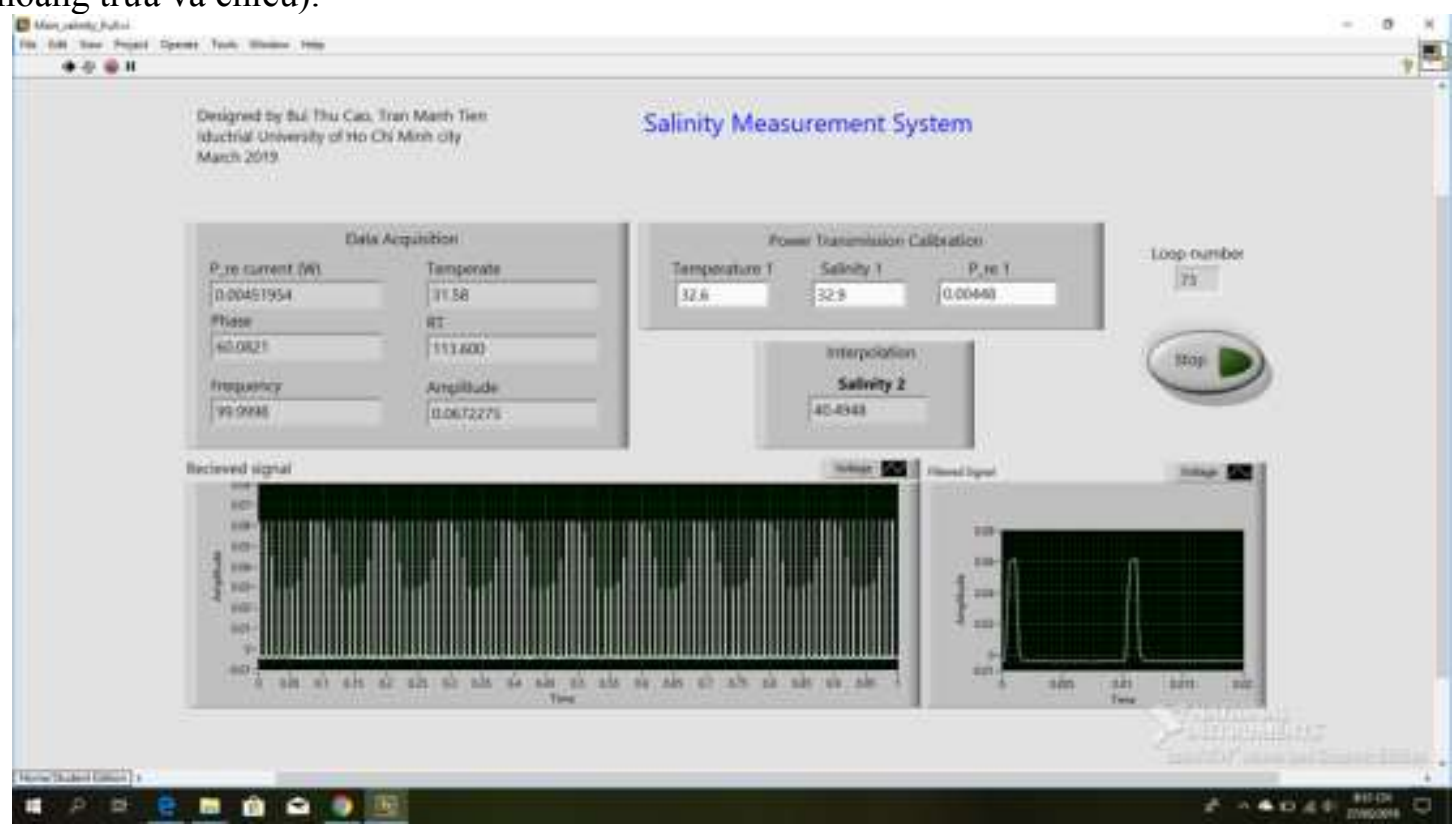

Hình 8. Giao diện phần mềm điều khiển sủ dụng Labview

Bảng 1 Dũ liệu thục nghiệm

\begin{tabular}{|r|r|r|r|r|r|l|}
\hline $\begin{array}{r}\text { Thời } \\
\text { gian }\end{array}$ & Nhiệt độ & \multicolumn{1}{|c|}{$\varepsilon_{r}$} & S\%o & S_EX\%o & Delta(S) & Ghi chú \\
\hline 9AM & 29.3 & 76.55 & 0.000 & 0.00 & 0.00 & $\begin{array}{l}\text { Hiệu chuấn công } \\
\text { suất }\end{array}$ \\
\hline 2PM & 32.1 & 75.44 & 1.210 & 3.650 & 2.44 & Lấy mẫu \\
\hline $4 \mathrm{PM}$ & 32.36 & 75.12 & 2.330 & 4.970 & 2.640 & Lấy mẫu \\
\hline 6PM & 31.7 & 75.03 & 3.520 & 6.120 & 2.600 & Lấy mẫu \\
\hline 8PM & 30.15 & 75.16 & 4.780 & 6.870 & 2.090 & Lấy mẫu \\
\hline
\end{tabular}



ĐIỆN CỰC KHÔNG TIÊP XÚC

\begin{tabular}{|r|r|r|r|r|r|l|}
\hline $9 \mathrm{AM}$ & 28.7 & 75.33 & 6.130 & 6.620 & 0.490 & Lấy mẫu \\
\hline $11 \mathrm{AM}$ & 29.8 & 74.75 & 7.260 & 6.960 & -0.300 & Lấy mẫu \\
\hline $2 \mathrm{PM}$ & 31.8 & 73.95 & 8.450 & 10.230 & 1.780 & Lấy mẫu \\
\hline $4 \mathrm{PM}$ & 31.8 & 73.68 & 9.810 & 11.200 & 1.390 & Lấy mẫu \\
\hline $6 \mathrm{PM}$ & 31.8 & 73.61 & 10.120 & 12.400 & 2.280 & Lấy mẫu \\
\hline $8 \mathrm{PM}$ & 30.5 & 73.71 & 11,340 & 12,900 & 1,560 & Lấy mẫu \\
\hline $9 \mathrm{AM}$ & 29.15 & 73.66 & 13,540 & 13,200 & $-0,340$ & Lấy mẫu \\
\hline $11 \mathrm{AM}$ & 30.05 & 72.78 & 16,810 & 17,100 & 0,290 & Lấy mẫu \\
\hline $2 \mathrm{PM}$ & 31.2 & 71.95 & 19,750 & 22,300 & 2,550 & Lấy mẫu \\
\hline $4 \mathrm{PM}$ & 31.5 & 71.33 & 22,750 & 24,200 & 1,450 & Lấy mẫu \\
\hline $6 \mathrm{PM}$ & 30.74 & 70.52 & 28,230 & 29,800 & 1,570 & Lấy mẫu \\
\hline $8 \mathrm{PM}$ & 30.2 & 69.82 & 32,780 & 31,900 & $-0,880$ & Lấy mẫu \\
\hline $9 \mathrm{PM}$ & 29.43 & 69.49 & 35,450 & 36,700 & 1,250 & Lấy mẫu \\
\hline $11 \mathrm{AM}$ & 30.21 & 68.74 & 38,320 & 38,900 & 0,580 & Lấy mẫu \\
\hline $2 \mathrm{PM}$ & 32.61 & 67.59 & 41,670 & 43,300 & 1,630 & Lấy mẫu \\
\hline & & & \multicolumn{7}{|c|}{ Trung bình sai số } & \pm 1.2 & \\
\hline
\end{tabular}

Các nguyên nhân khách quan và chủ quan gây ra sai số của hệ thống được rút ra như sau:

○ Do ảnh hưởng của hiện tượng fading sóng trong quá trình lan truyền. Sóng từ anten phát tới anten thu từ nhiều hướng: truyền thẳng và tán xạ từ thành bể và từ môi trường ngoài. Điều này làm thay đổi công suất thu, gây ra sai số cho phép đo.

○ Do ảnh hưởng của nhiễu nhiệt lên các linh kiện bán dẫn, làm thay đổi hệ số khuếch đại, dẫn đến làm thay đỗi công suất thu. Việc thay đổi công suất thu gây ra sai số cho phép đo.

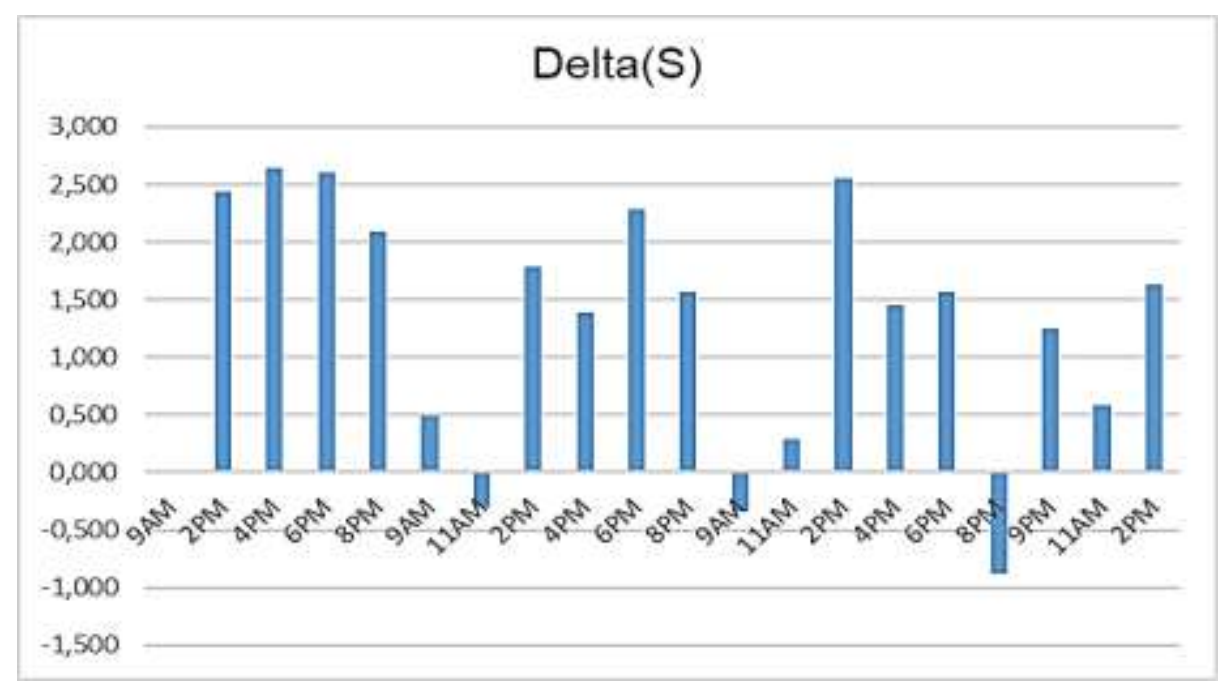

Hình 9. Thông kế sai số của độ mặn của hệ thống so với thiết bị chuẩn 
ĐIỆN CỰC KHÔNG TIÊP XÚC

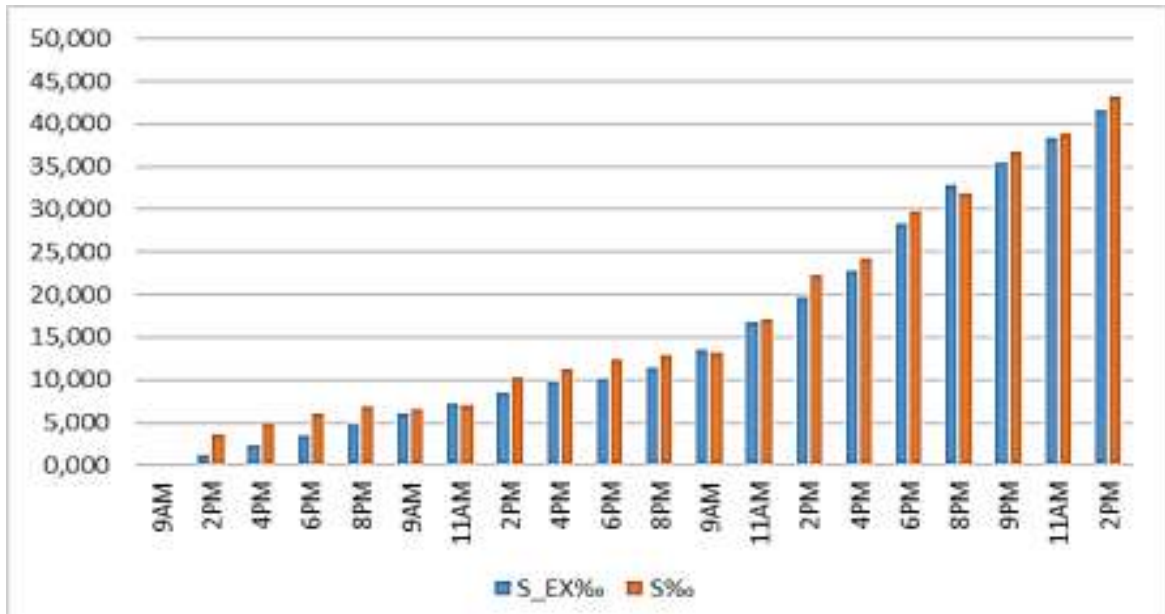

Hình 10. Minh họa các giá trị độ mặn đo bằng phuơng pháp đề nghị (S\%o) và đo bằng máy đo chuyên dụng độ dẫn EC170 của EXTECH (S_EX\%)

\section{CONCLUSION}

Bài báo này đã trình bày một phương pháp mới trong thiết kế bộ cảm biến đo độ mặn bằng phương pháp điện cực không tiếp xúc. Bắt đầu bằng việc phân tích và xây dựng hàm toán học mô tả mối liên hệ giữa công suất truyền dẫn sóng radio với hằng số điện môi của trường và mối liên hệ giữa hằng số điện môi của môi trường với độ mặn của nước, tác giả đã đưa ra mô hình thiết kế hệ thống thiết bị đo độ mặn. Từ mô hình thiết kế, tác giả đã đưa ra các thiết kế chi tiết phù hợp cho từng khối chức năng.

Kết quả thực nghiệm cho thấy phép đo cho sai số trung bình không quá $1.2 \%$ so với kết quả đo của máy đo độ mặn kĩ thuật số chuẩn, EC170 của hãng EXTECH. Nếu so sánh với các máy đo hiện tại thì thiết bị đo được thiết kế có độ chính xác thấp. Tuy nhiên nếu so sánh về hiệu quả ứng dụng đo online bền bỉ liên tục 24/24 trong thời gian hàng tháng trời thì khả năng đáp ứng của thiết bị được thiết kế là tuyệt vời và không có thiết bị đo nào trên thị trường có thể làm được tại thời điểm hiện nay. Hơn nữa, nếu xét về góc độ ứng dụng trong nuôi trồng thủy sản, ví dụ tôm/ cá nước lợ sống trong môi trường độ mặn có phạm vi từ $5-40 \%$, thì phương pháp đo được đề nghị cho sai số trong khoảng $1 \div 2 \%$ là chấp nhận được.

Một số điểm đóng góp mới và nổi bật của bài báo:

○ Sử dụng bức xạ radio để đo hằng số điện môi, từ đó là cơ sở để giải bài toán giúp nội suy ra thông số độ mặn của nước.

○ Kỹ thuật dùng bức xạ radio để đo hằng số điện môi có thể được mở rộng ứng dụng để đo nồng độ vật chất của rất nhiều loại vật liệu khác nhau, như đo lưu lượng khí gas hay đo lưu lượng chất lỏng.

- Đóng góp quan trọng nhất của bài báo này là đề xuất phương pháp đo độ mặn bằng điện cực không tiếp, nó giúp tạo ra thiết bị đo có thể đo online có độ bền, độ ổn định cao, mà không cần phải vệ sinh đầu đò theo thời gian.

\section{REFERENCES}

[1] Misco, "http://www.misco.com/Downloads/MISCO-TB-SEAWATER.pdf," Ohio, USA, 2007.

[2] R. W. Austin and G. Halika!5, "THE INDEX OF REFRACTION OF SEAWATER," U.S. DEPARTMENT OF COMMERCE, National Technical Information Service, Virginia, US, 1976.

[3] B. Sklar and P. K. Ray, in Digital Communications: Fundamentals and Applications, 2014, pp. 242-256.

[4] J. D. Kraus, Antennas, Ohio, US: Tata McGraw-Hill, 2001.

[5] A. Q. Jakhrani, S. R. Samo, H. Sobuz, M. A. Uddin and M. J. Ahsan, "Assessment of Dissolved Salts 
Concentration of Seawater in the Vicinity of Karachi," International Journal of Structural and Civil Engineering, vol. 1, no. 2, pp. 61-69, 2012.

[6] "http://www.horiba.com/fileadmin/uploads/Scientific/water_quality/Documents/Application_Notes/HIS/20__Determination_Of_Potassium_In_Sea_Water.pdf," Horiba.

[7] L. A. KLEIN and. C. T. SWIFT, "An Improved Model for- the Dielectric Constant of Sea Water at Microwave Frequencies," IEEE TRANSACTIONS ON ANTENNAS AND PROPAGATION, vol. Vol.AP 25, no. No1, pp. 104-111, 1977.

[8] R. Somaraju and J. Trumpf, "Frequency, temperature and salinity variation of the permittivity of Seawater," IEEE Transactions on Antennas and Propagation, vol. 54, no. 11, pp. 3441 - 3448, 2006.

[9] T. Meissner and F. Wentz, "The Complex Dielectric Constant of Pure and Sea Water from microwave satellite observations," IEEE Transactions on Geoscience and Remote Sensing, vol. 42, no. 9, pp. 1836-1849, 2004.

[10] D. H. Gadani, V. A. Rana, S. P. Bhatnagar, A. N. Prajapati and A. D. Vyas, "Effect of Salinity on the Dielectric Properties of Water," Indian Journal of Pure \& Applied Physics.

Ngày nhận bài:08/04/2019

Ngày chấp nhận đăng:14/05/2019 